\title{
Efficacy of combining oral Chinese herbal medicine and NB-UVB in treating psoriasis vulgaris: a systematic review and meta-analysis
}

Lihong Yang 1,3,4, Claire Shuiqing Zhang ${ }^{5}$, Brian May ${ }^{5}$, Jingjie Yu², Xinfeng Guo 1,3,4, Anthony Lin Zhang ${ }^{5}$, Charlie Changli Xue ${ }^{3,4,5^{*}}$ and Chuanjian Lu $2,3,45^{*}$

\begin{abstract}
Background: The combination of a Chinese herbal medicine (CHM) bath and narrowband ultraviolet B (NB-UVB) improved the efficacy of NB-UVB treatment of psoriasis vulgaris, but bath therapy is inconvenient. Oral CHM plus NBUVB has been tested in clinical practice. This study aims to evaluate whether adding oral CHM could be beneficial for NB-UVB therapy by a systematic review and meta-analysis.

Methods: Nine English and Chinese databases were searched from their inception to April 2014. Randomized controlled trials (RCTs) comparing the combination of orally administered CHM and NB-UVB with that of CHM placebo and NB-UVB or NB-UVB alone for treating psoriasis vulgaris and reporting Psoriasis Area Severity Index (PASI) outcomes were included. A systematic review, meta-analysis, risk of bias assessment and the GRADE assessment were conducted in accordance with Cochrane Collaboration methodology to assess the evidence for efficacy outcome. Data were analyzed in RevMan5.2.

Results: Eighteen eligible RCTs ( $n=1416)$ were included for systematic review, and $17(n=1342)$ of them were included in meta-analysis. Risk of bias in terms of blinding was high and so was in publication bias. Quality of evidence was low according the GRADE assessment. PASI-60 or above [risk ratio (RR) $=1.35,95 \%$ confidence interval (Cl) $1.26-1.45, I^{2}=5 \%$, number needed to treat $=4.27$ ] and PASI-90 or above $\left(\mathrm{RR}=1.71,95 \% \mathrm{Cl} 1.45-2.01, \mathrm{I}^{2}=0 \%\right.$, number needed to treat $=5.92$ ) were higher in the intervention group. The combination treatment conferred a $24 \%$ benefit of PASI-60 or above ( 83 vs $59 \%, \mathrm{RR}=1.35,95 \% \mathrm{Cl} 1.26-1.45, P<0.01$ ). The incidence of NB-UVB-induced adverse events was lower in the intervention group (95/464 vs 123/428, $\mathrm{RR}=0.66,95 \% \mathrm{Cl} 0.46-0.96, P<0.01$ ). Mild gastrointestinal reactions (2.87 \%) and liver function impairments (0.68\%) were reported in the intervention group. No serious adverse events were reported.
\end{abstract}

Conclusion: The orally administrated CHM combined with NB-UVB in treating psoriasis vulgaris showed improved efficacy but quality of evidence was low.

\footnotetext{
*Correspondence: charlie.xue@rmit.edu.au; luchuanjian888@vip.sina.com

${ }^{4}$ Guangdong Provincial Academy of Chinese Medical Sciences,

Guangzhou 510120, China

${ }^{5}$ China-Australia International Research Centre for Chinese Medicine, School of Health Sciences, RMIT Health Innovations Research Institute, RMIT University, PO Box 71, Bundoora, Melbourne, VIC 3083, Australia Full list of author information is available at the end of the article
} provided you give appropriate credit to the original author(s) and the source, provide a link to the Creative Commons license, and indicate if changes were made. The Creative Commons Public Domain Dedication waiver (http://creativecommons.org/ publicdomain/zero/1.0/) applies to the data made available in this article, unless otherwise stated. 


\section{Background}

Psoriasis is a common, chronic and recurrent inflammatory skin disease with a global prevalence of 3-4\% [1]. The most common form, psoriasis vulgaris, affects 80-90 \% of patients with psoriasis [2]. Ultraviolet-based therapies including psoralen plus ultraviolet A (PUVA), narrowband ultraviolet $B(N B-U V B)$ and broad-band ultraviolet $\mathrm{B}$ (BB-UVB) are effective treatments for psoriasis vulgaris. There is an increased risk of developing skin cancer following PUVA therapy $[3,4]$, although NB-UVB is more effective than BB-UVB and less effective than PUVA. Therefore, NB-UVB is preferred in the clinical management of psoriasis vulgaris $[3,5]$. Because the clearance rate of NBUVB treatment was reported in 51-75\% [6], adjuvant treatments are used to improve the effectiveness. Chinese herbal medicine (CHM) has been used for treating psoriasis vulgaris in China. The combination of a CHM bath and NB-UVB was beneficial in psoriasis vulgaris patients [7], but this approach has limitations in practice because a 30-min bath was required prior to each NB-UVB session. Therefore, the combination of oral CHM and NB-UVB has been used in clinical practice for treating psoriasis vulgaris [8]. However, it is still unclear if oral CHM combined with NB-UVB is beneficial for psoriasis vulgaris treatment.

This study aims to evaluate whether oral CHM could provide an added beneficial effect on NB-UVB therapy by a systematic review and meta-analysis.

\section{Methods}

\section{Search strategies}

Nine English and Chinese electronic databases, namely, the PubMed, Embase, CINAHL, CENTRAL, AMED, China National Knowledge Infrastructure (CNKI), Chinese BioMedical Literature (CBM), Chinese Science Journals Full Text (CQVIP) and Wanfang databases, were searched from their inceptions to April 2014. Search terms were grouped in three categories: (1) condition (psoriasis); (2) intervention (CHM, acupuncture, moxibustion, and other traditional Chinese medicine therapies); and (3) study type (randomized controlled trials), adjusted for each database (Table 1). The cited references of review articles were searched to identify additional studies.

\section{Study selection criteria}

Randomized controlled trials (RCT) that compared orally administered CHM plus NB-UVB with CHM placebo plus NB-UVB or NB-UVB alone for psoriasis vulgaris were selected. The Psoriasis Area Severity Index (PASI) was used in clinical trials for outcome measurement following psoriasis treatment. The PASI measures the redness, thickness and scaling of the lesions and the area of involvement, with a total score ranging from 0 to 72 . Reduction of disease severity was assessed based on the
Table 1 Search strategy in PubMed

\begin{tabular}{|c|c|}
\hline Categories & Search terms \\
\hline \multirow[t]{8}{*}{ Condition } & 1. Skin diseases, papulosquamous (MeSH) \\
\hline & 2. Psoriasis (MeSH) \\
\hline & 3. Psoriases \\
\hline & 4. Pustulosis palmaris et plantaris \\
\hline & 5. Palmoplantaris pustulosis \\
\hline & 6. Pustular psoriasis of palms and soles \\
\hline & 7. Psoria ${ }^{a}$ \\
\hline & 8. \#1-\#7/OR \\
\hline \multirow[t]{25}{*}{ Intervention } & 9. Medicine, Chinese traditional (MeSH) \\
\hline & 10. Chinese traditional medicine \\
\hline & 11. Chinese herbal drugs \\
\hline & 12. Chinese drugs, plant \\
\hline & 13. Medicine, traditional \\
\hline & 14. Ethnopharmacology \\
\hline & 15. Ethnomedicine \\
\hline & 16. Ethnobotany \\
\hline & 17. Medicine, Kampo \\
\hline & 18. Kampo \\
\hline & 19. TCM \\
\hline & 20. TCM \\
\hline & 21. Medicine, ayurvedic ${ }^{b}$ \\
\hline & 22. Phytotherapy \\
\hline & 23. Herbology \\
\hline & 24. Plants, medicinal \\
\hline & 25. Plant preparations \\
\hline & 26. Plant extracts \\
\hline & 27. Plants, medicine \\
\hline & 28. Materia medica \\
\hline & 29. Single prescription \\
\hline & 30. Chinese medicine herb \\
\hline & 31. Herbal medicine \\
\hline & 32. Herbs \\
\hline & 33. \#9-\#32/OR \\
\hline \multirow[t]{9}{*}{ Study type } & 34. Randomized controlled trial (pt) \\
\hline & 35. Controlled clinical trial (pt) \\
\hline & 36. Randomized (tiab) \\
\hline & 37. Randomly (tiab) \\
\hline & 38. Placebo (tiab) \\
\hline & 39. Trial (tiab) \\
\hline & 40. Groups (tiab) \\
\hline & 41. \#34-\#40/OR \\
\hline & 42. \#8 AND \#33 AND \#41 \\
\hline
\end{tabular}

\footnotetext{
any word starts from Psoria

b Traditional Indian Herbal Medicine
}

percent change in PASI score. PASI-75, referring to a $75 \%$ reduction in PASI, was considered effective in psoriasis treatment [6]. Only studies reporting PASI scores as an outcome were included. 
Studies were excluded if they met the following exclusion criteria: (1) participants had comorbidities; (2) CHM products included Western pharmaceuticals; and (3) there were co-interventions that used anti-psoriatic drugs or Chinese medicine treatments other than oral CHM.

\section{Data extraction and quality assessment}

Two authors (LY, CZ) independently extracted and crosschecked data using a pre-defined sheet including: study location, setting, participants, sample size, treatment duration, intervention details, dropouts, outcome measures and adverse events (AE). If data were incomplete or appeared incorrect, attempts were made to contact the authors to request additional information or clarifying data. LY and CZ conducted risk of bias assessments independently using the Cochrane Collaboration tool for assessing risk of bias [9]. The grades of recommendation, assessment, development and evaluation (GRADE) approach was used to assess the quality of the evidence for individual outcomes, considering risk of bias, heterogeneity, directness of evidence, precision of effect estimation, and risk of publication bias. Disagreements were resolved by discussion with the two other authors (XG, AZ).

\section{Data analysis}

Meta-analysis was performed in RevMan5.2. Risk ratios (RR) with $95 \%$ confidence intervals (CI) for dichotomous data and mean differences (MD) with $95 \%$ CIs for continuous data were reported. Data were pooled to estimate the effect size by the random-effects model [9]. Subgroup analysis was performed according to treatment duration, baseline severity, the CHM formulas and main formula ingredients. Sensitivity analysis was performed taking account of the risk of bias to assess the reliability of the pooled results. Dropouts or withdrawals were considered ineffective cases in an intention-to-treat (ITT) analysis to obtain a conservative estimation. Publication bias was assessed by funnel plots and Egger's linear regression test. An asymmetrical funnel plot or $P<0.05$ in Egger's test indicated a publication bias.

\section{Results}

The initial searches yielded 5782 articles. After removing duplicates and screening titles and abstracts, 576 fulltext articles were retrieved for further judgment. Eighteen articles were included [8, 10-26]. The study selection process is presented in Fig. 1.

\section{Description of the included studies}

The 18 included trials, involving 1416 participants with psoriasis vulgaris, were conducted in hospitals in China and published in Chinese from 2004 to 2014. Characteristics of the included studies are summarized in Table 2. All included studies evaluated oral CHM added to NBUVB vs NB-UVB alone, and six $[8,13,14,18,19,22,24]$ were three-arm trials that included a comparison with oral CHM alone (not analyzed in this study). A placebo was not used in any included study. Two studies used disease severity as one of the inclusion criteria. Fu et al. [11] recruited psoriasis vulgaris participants with an affected body surface area (BSA) $>10 \%$ and Sheng et al. [19] recruited psoriasis vulgaris participants with an affected BSA $>10 \%$ and PASI score $\geq 10$. Ten studies reported mean PASI score at baseline. The mean PASI scores were $<10$ in two studies [10,17], $>20$ in one study [16], and ranged from 14.32 to 19.19 in the remaining seven studies [8, 14, 15, 18, 19, 22, 26]. Eight studies recruited psoriasis vulgaris participants targeting specific Chinese medicine patterns $[8,10,15,16,21,22,25,26]$.

\section{Interventions}

All studies reported that NB-UVB phototherapy was consistently prescribed to intervention groups and control groups. However, there was variation across studies in terms of the initial dosage, dosage increment regimen, treatment frequency and total number of treatment sessions. Most of the studies applied phototherapy two to three times per week for 10-29 treatment sessions in total.

Huang et al. [12] used a single herb extract product; the other 17 studies used multi-ingredient formulas in decoctions $[10,11,13,16,18,19,21,25]$, pills $[12,17,24]$, capsules [20], or granules [14, 22, 26]. CHM was administered daily. No two studies used an identical herbal formula. Although all formulas varied across studies (Table 2), there were similarities in the herb ingredients used. The most frequently used herbs were Rehmannia glutinosa (sheng di huang) in 13 studies [8, 11, 13-15, 18-24, 26], Angelica sinensis (dang gui) in 11 studies [10, 11, 13-15, 19, 20, 23, 24, 26], Smilax glabra (tu fu ling) in nine studies [8, 10, 13, 15, 17, 19, 22, 23, 26], Salvia miltiorrhiza (dan shen) in eight studies [10, 11, 15, 16, 22-24], and Glycyrrhiza uralensis (gan cao) in eight studies [10, 11, 13, 18, 19, 21, 24, 26]. Furthermore, three herbs, $R$. glutinosa, A. sinensis, and S. glabra, were used together in six studies $[8,13,15,19$, $23,26]$. The details of formula and herbs used by included studies can be found in Additional file 1: Table S1.

\section{Treatment and follow-up durations}

Sun et al. [20] only reported the total number of phototherapy treatment sessions. The treatment durations of the other 17 studies ranged from 4 to 12 weeks, and ten studies were of 8 weeks ( 2 months).

Seven studies included follow up with participants after treatment. Four studies followed all included participants 


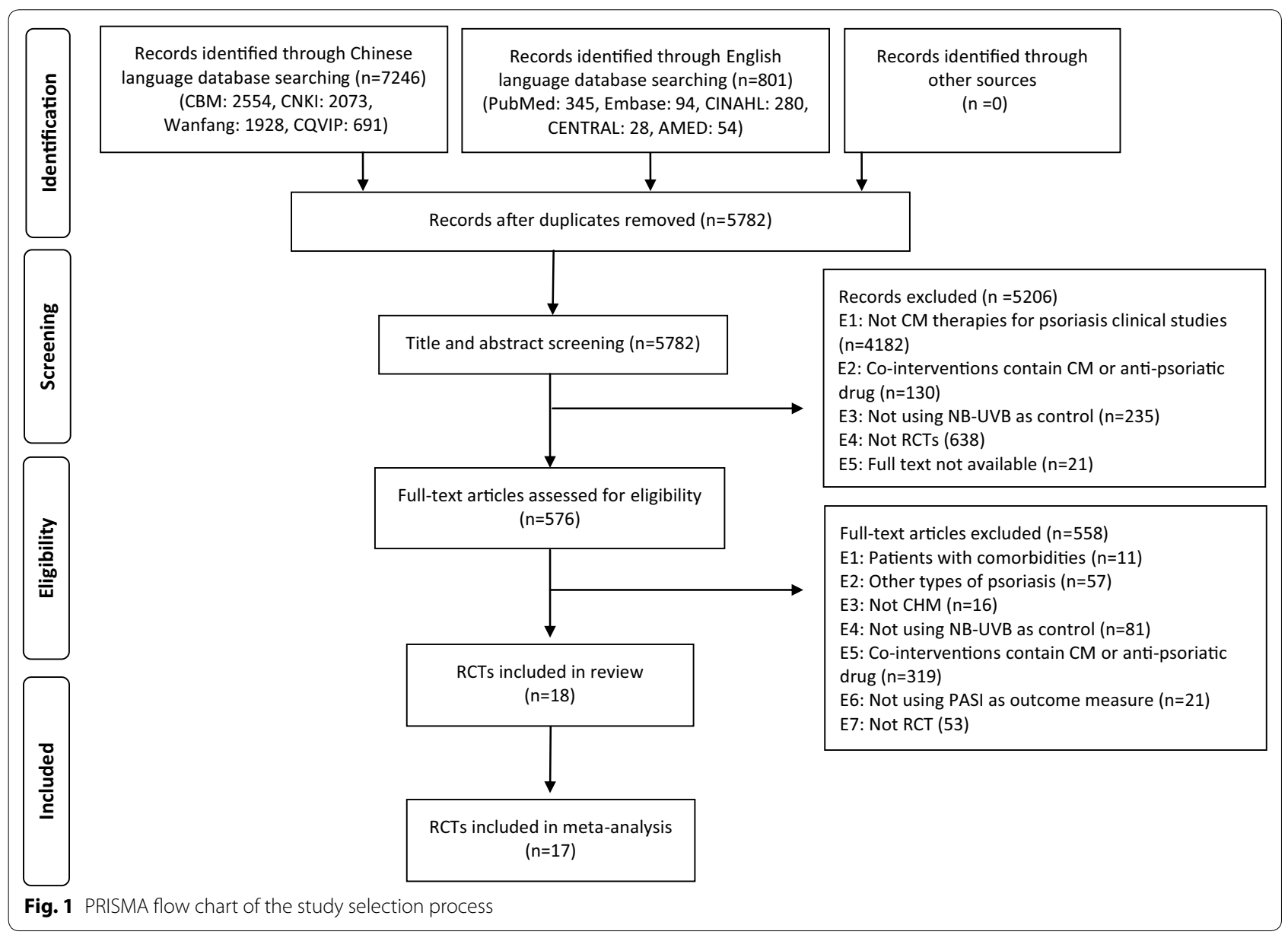

for 3 months [15], 6 months [22, 23], and 1 year [25]. Three studies followed participants who achieved PASI90 for 6 months $[10,18]$ and 1 year [15].

\section{Outcome measures}

Seventeen of the 18 studies reported the numbers of participants who achieved PASI-95 or PASI-90, and PASI-60 (at least 95, 90, 60 \% reduction in PASI), with reference to the Consensus of Diagnosis and Treatment of Psoriasis Vulgaris with Integrative Medicine [27]. One study reported PASI-95 and PASI-70 [16]. As PASI-50 was taken as the minimum clinically meaningful improvement $[6,28]$, PASI-60 and PASI-70 were conservatively pooled as PASI-60 to analyze the effect of the combination of oral CHM and NB-UVB. Four studies reported the relapse rates at the end of the follow-up [10, 11, 18, $23]$, but the definitions of relapse were not stated.

\section{Dropouts and withdrawals}

Liu and Sheng [13] reported that one participant in the treatment group and two in the control group were excluded after randomization because of comorbidities, and there were five dropouts in the treatment group and four dropouts in the control group for low compliance. Yan et al. [24] reported one withdrawal in the NB-UVB group owing to AEs, but a phototoxicity reaction was not observed. No dropout was reported in the other 16 studies.

\section{Overall efficacy}

One study was not included in the meta-analysis, because the outcome measures were PASI-25 and PASI-10 [25]. This study reported that the effect rate was much higher in the combination group than in the NB-UVB group in terms of PASI-25 (78.4 vs $8.1 \%$ ). The results of the remaining 17 studies were pooled to estimate the efficacy of the combination treatment.

\section{PASI-60 and above}

The mean percentage of participants who achieved PASI60 at the end of treatment was $83 \%$ in the combination group and $59 \%$ in the NB-UVB group. PASI-60 was significantly higher in the combination group than in the NB-UVB group [Fig. 2, 17 studies, $R R=1.35,95 \% \mathrm{CI}$ 


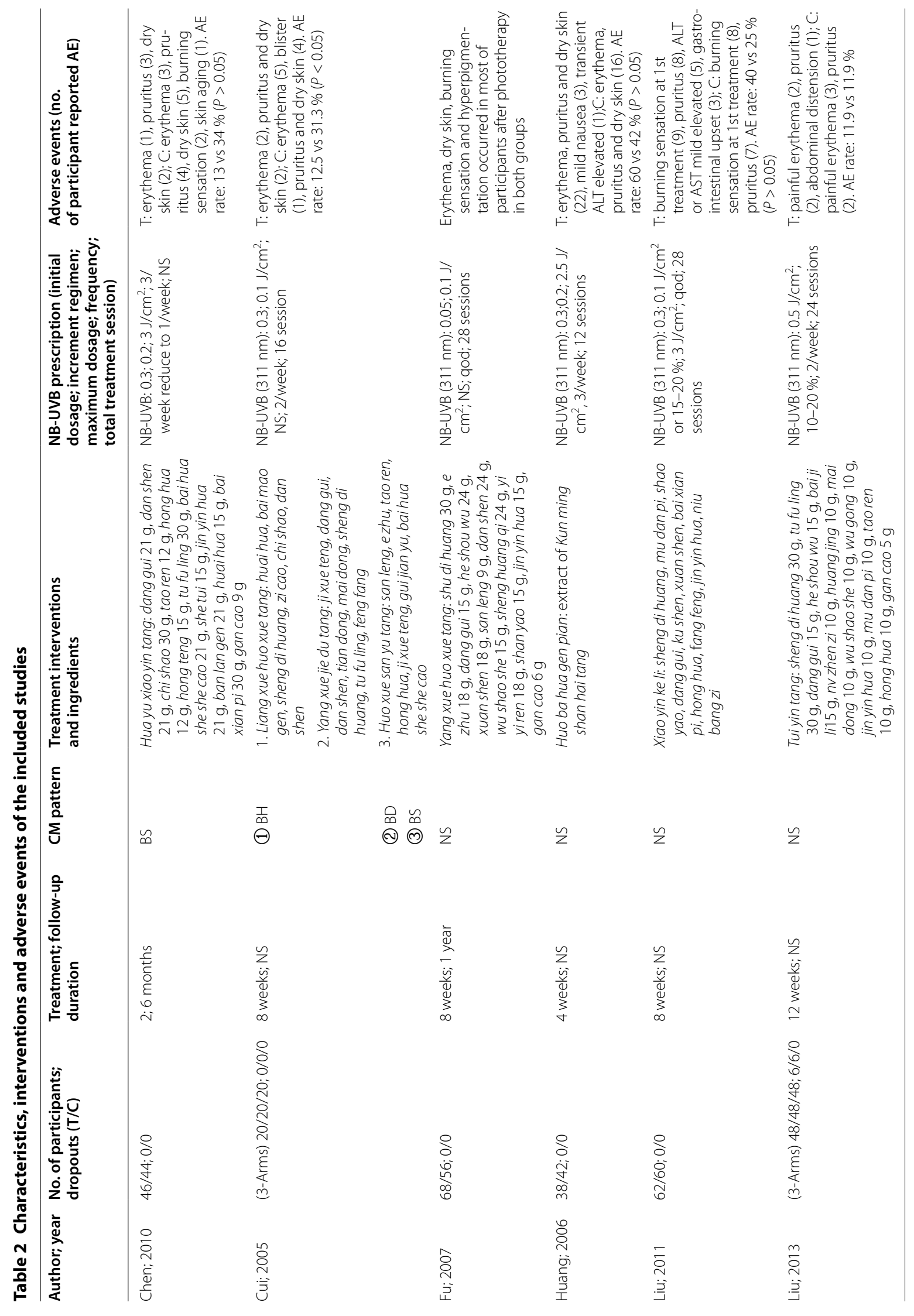




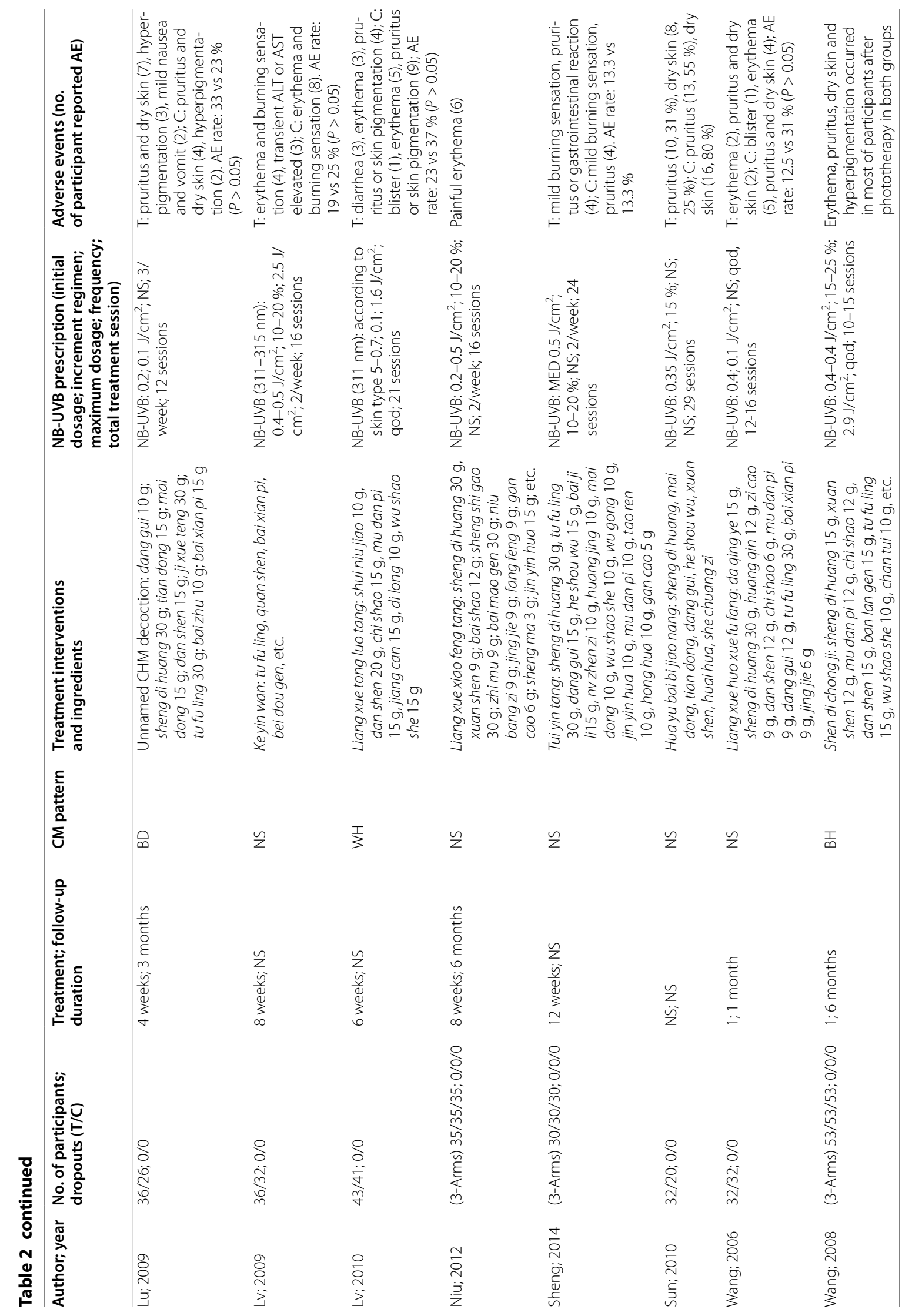




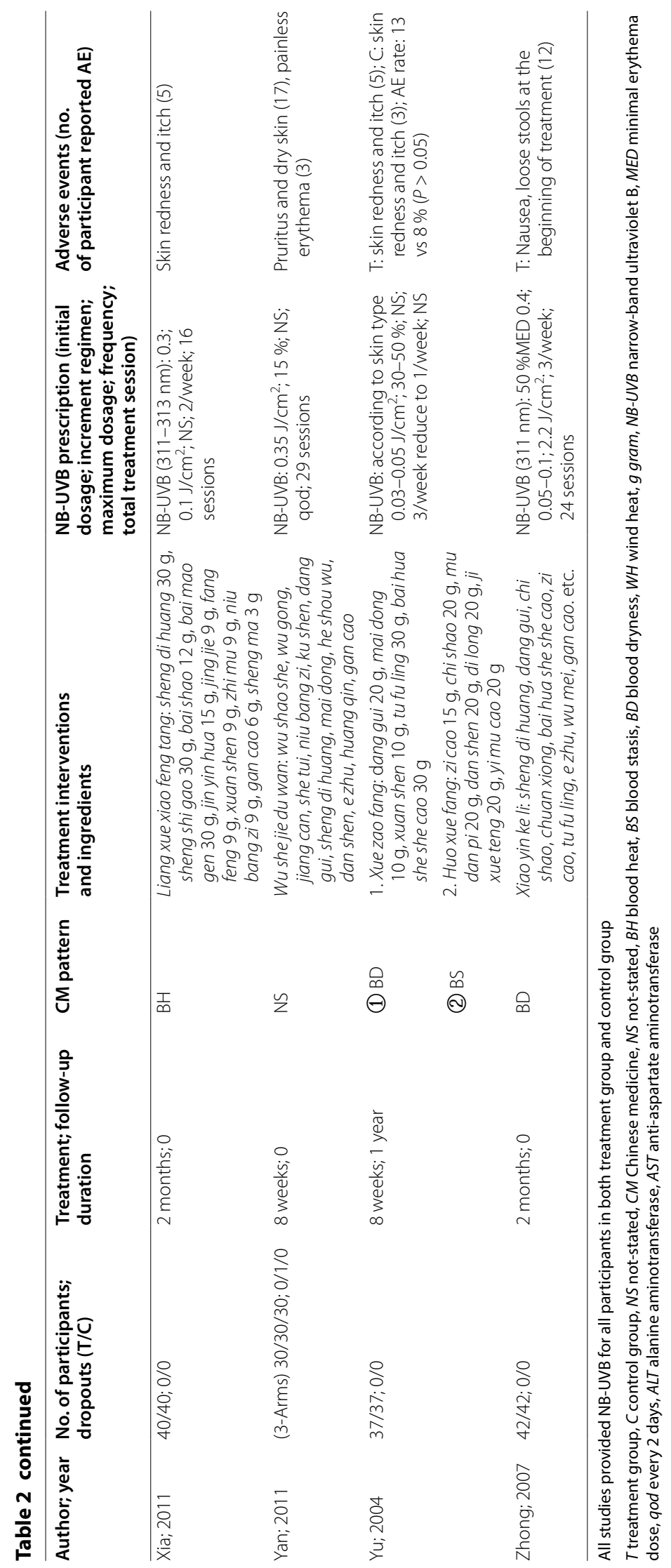




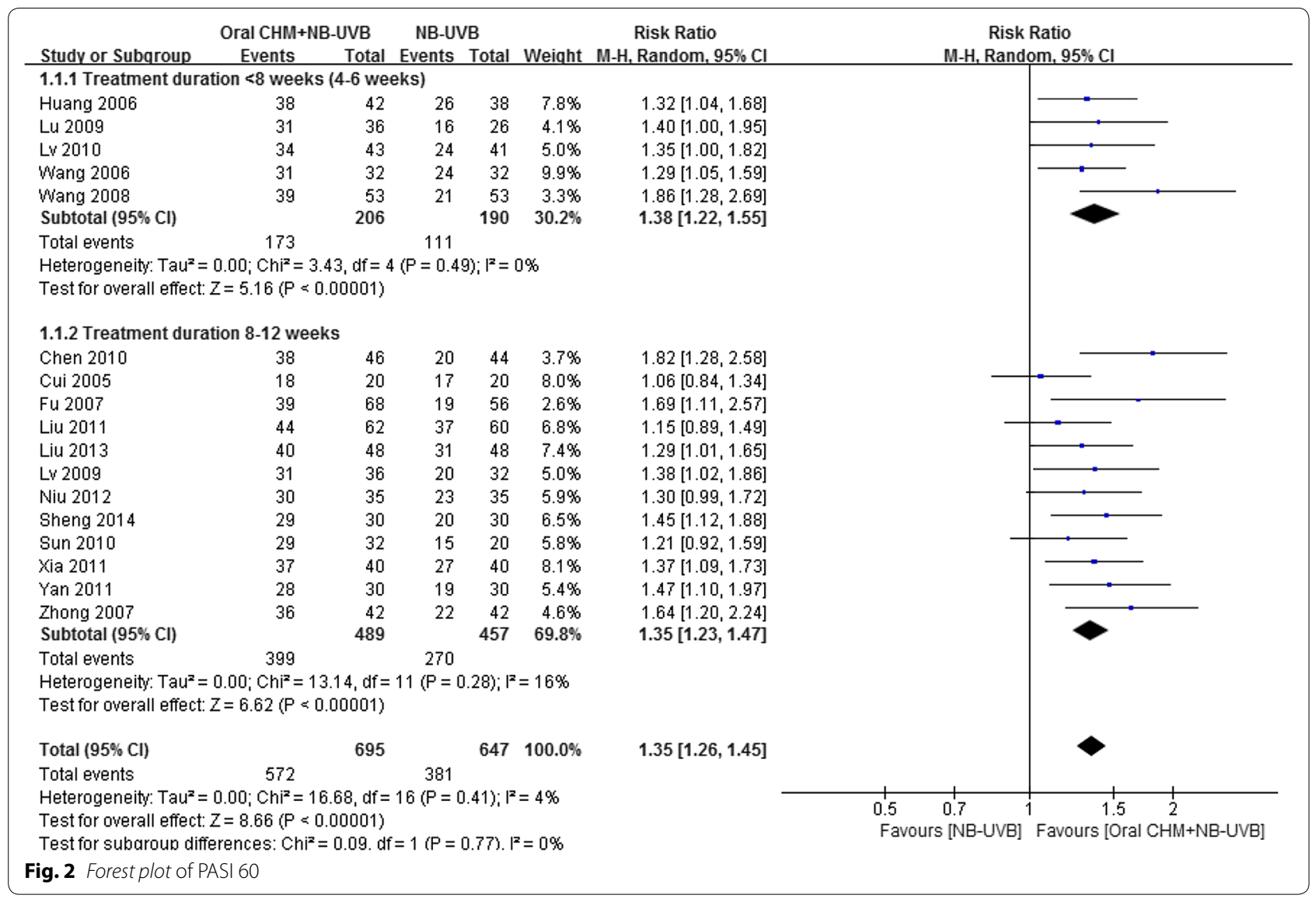

1.26-1.45, $P<0.01, \mathrm{I}^{2}=5 \%$; number needed to treat $(\mathrm{NNT})=4.27]$.

The most common treatment duration of the included studies was 2 months. Subgroup analysis showed that the pooled results were consistent between studies with 4-6 weeks treatment (five studies, $\mathrm{RR}=1.38$, $95 \%$ CI 1.22-1.55, $\left.P<0.01, \mathrm{I}^{2}=0 \%\right)$ and studies with 8-12 weeks treatment ( 12 studies, $\mathrm{RR}=1.34,95 \% \mathrm{CI}$ $\left.1.23-1.47, P<0.01, \mathrm{I}^{2}=16 \%\right)$. For the seven studies that included participants with moderate to severe psoriasis, the result for PASI-60 was similar to that for the total pool (RR 1.35, $95 \%$ CI 1.17-1.56, $P<0.01, \mathrm{I}^{2}=45 \%$ ).

The most common herb combination used was $R$. glutinosa, A. sinensis and S. glabra. When the studies using this herb combination were pooled, the result was consistent with the result of the total pool (Table 3), but with moderate heterogeneity (six studies, $\mathrm{RR}=1.31$, $95 \%$ CI $\left.1.16-1.54, P<0.01, \mathrm{I}^{2}=22 \%\right)$. Of these six studies, one study [8] prescribed three different formulas targeting three different Chinese medicine patterns, and the above three-herb combination was only included in one of the formulas. However, PASI-60 was not reported separately according to the different formulas. Therefore, the data reported did not represent the outcome of the particular formula which contained the three common herbs. When this study was excluded, the result showed a superior effect for the combination treatment without heterogeneity $(\mathrm{RR}=1.37,95 \% \mathrm{CI} 1.22-1.54, P<0.01$, $\left.\mathrm{I}^{2}=0 \%\right)$.

For the five studies judged low risk on sequence generation, the result $(\mathrm{RR}=1.39,95 \% \mathrm{CI} 1.23-1.58, P<0.01$, $\mathrm{I}^{2}=0 \%$ ) was consistent with that for the total pool.

\section{PASI-90}

The meta-analysis of PASI-90 or above also favored the combination group (Fig. 3, 17 studies, $\mathrm{RR}=1.71,95 \% \mathrm{CI}$ $1.45-2.01, P<0.01, \mathrm{I}^{2}=0 \%$;NT $\left.=5.92\right)$.

\section{Post-follow-up relapse rate}

The mean relapse rate of the three studies that followed participants who achieved PASI-90 was $11 \%$ in the combination group and $60 \%$ in the NB-UVB group 3 months-1 year after treatment. Because the definition of relapse was unclear and there were different follow up durations, meta-analysis of these studies was not performed. 
Table 3 Results of meta-analysis

\begin{tabular}{|c|c|}
\hline Outcomes & $\mathrm{RR}(95 \% \mathrm{Cl}), \mathrm{I}^{2}, \mathrm{NNT}$ \\
\hline \multicolumn{2}{|l|}{ PASI-60 or above } \\
\hline 17 studies & $1.35(1.26,1.45), I^{2}=5 \%, 4.27$ \\
\hline Studies with <8 (4-6) weeks treatment (five studies) & $1.38(1.22,1.55), I^{2}=0 \%$ \\
\hline Studies with 8-12 weeks treatment (12 studies) & $1.34(1.23,1.47), I^{2}=16 \%$ \\
\hline Studies with moderate to severe psoriasis (baseline PASI 14-20) (seven studies) & $1.35(1.17,1.56), I^{2}=45 \%$ \\
\hline Studies using three common herbs (di huang, dang gui, tu fu ling) (six studies) & $1.31(1.16,1.47),\left.\right|^{2}=22 \%$ \\
\hline Studies using three common herbs (five studies, one study that used three formulae was excluded) & $1.37(1.22,1.54), I^{2}=0 \%$ \\
\hline Studies with low risk on sequence generation (five studies) & $1.39(1.23,1.58), I^{2}=0 \%$ \\
\hline \multicolumn{2}{|l|}{ PASI-90 or above } \\
\hline 17 studies & $1.71(1.45,2.01), 1^{2}=0 \%, 5.92$ \\
\hline \multicolumn{2}{|l|}{ NB-UVB-induced AEs } \\
\hline 12 studies & $0.66(0.46,0.96), I^{2}=53 \%$ \\
\hline Studies with <8 (4-6) weeks treatment (four studies) & $0.77(0.42,1.43), 1^{2}=62 \%$ \\
\hline Studies with 8-12 weeks treatment (eight studies) & $0.60(0.38,0.94), I^{2}=46 \%$ \\
\hline
\end{tabular}

$R R$ RISK ratio, NNT number need to treat

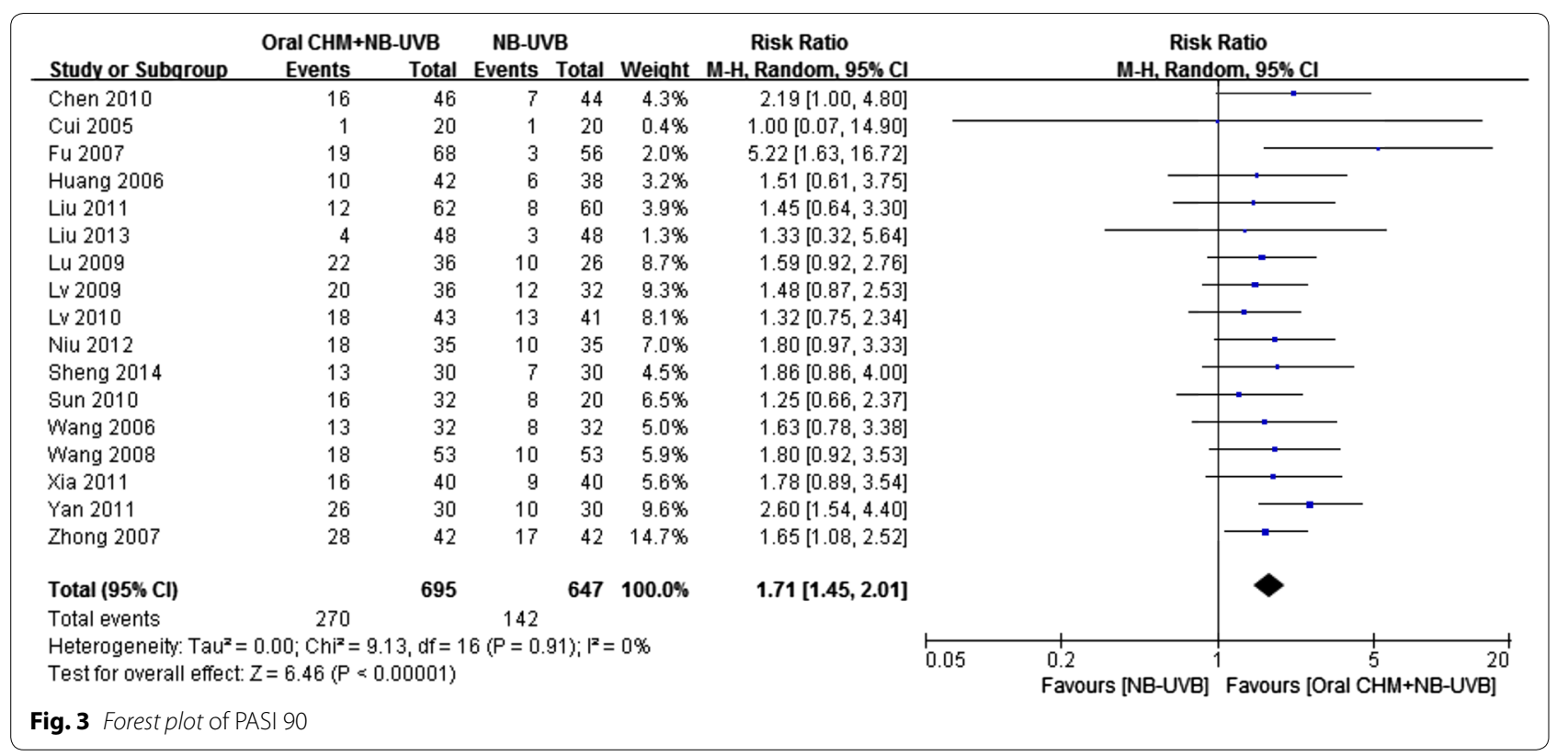

\section{Adverse events}

All studies reported AEs (Table 2). Zhong et al. [26] only reported AEs in the combination group, and three studies $[18,21,24]$ reported skin reactions but did not mention which group these occurred in. One participant withdrew because of a phototherapy AE [24]; the other withdrawals were not associated with AEs. All AEs reported in the NB-UVB groups were skin reactions, with a mean rate of $31.58 \%(8-80 \%)$. AEs reported in the combination groups were skin reactions, gastrointestinal reactions and elevated liver enzymes. The total AE rate $(24.42 \%)$ in the combination groups was a little lower than that in NBUVB groups. In addition, combination treatment lowered the incidence rate of skin reactions including pruritus, skin dryness, erythema, blistering and hyperpigmentation [95/464 (20.47\%) vs 123/428 (28.74\%); RR = 0.66, $95 \%$ CI 0.46-0.96]. The subgroup analysis showed a significant difference in studies administering 8-12 weeks of treatment (eight studies, $\mathrm{RR}=0.60,95 \%$ CI 0.38-0.94), but not in studies with a duration of 4-6 weeks (four studies, RR $=0.77,95 \%$ CI 0.42-1.43). Mild gastrointestinal reactions, such as nausea, vomiting, abdominal 
distension and stool change, were reported in the combination groups $(21 / 732,2.87 \%)[12-14,16,17,26]$. Five cases of elevated liver enzymes were reported (5/732, $0.68 \%)$. One case returned to normal within 1 month without additional treatment [12], three returned to normal by reducing the dosage of the CHMs [17], and the outcome was not reported for one [14]. The relationships between the AEs and the interventions were not further discussed in any study. No severe AE was reported.

\section{Risk of bias assessment}

Risk of bias assessments are presented in Fig. 4. Attempts were made to contact the original authors to obtain further information on 13 studies but the authors of 12 studies could not be reached and the author of one study refused to provide the further information we requested; therefore, risk of bias assessments were based on the published texts. For "sequence generation", five studies that used random number tables (a table of random numbers is available in statistics textbooks or generated by computer) $[14,16,17]$ and drawing lots (including cointossing, dice-throwing, or card-shuffling) [13, 19] were assessed as "low risk", and the others were assessed as "unclear". All studies were assessed as "unclear" for "allocation concealment" owing to the lack of information. All studies were assessed as "high risk" for "blinding of participants and research personnel", because all studies used an add-on therapy and a placebo was not used for the CHM in any study. The blinding of outcome assessors was judged "unclear" because information was not provided. One study [24] had one withdrawal in the NB-UVB group, and it was assessed as "low risk" for "incomplete outcome data", because the proportion of missing data was not enough to have an impact on the effect estimate. The amount of missing outcome data in one study [13] was $12.5 \%$ and ITT analysis was not performed, so this study was assessed as "high risk" for "incomplete outcome data". The others were assessed as "unclear". All studies were "low risk" for "selective reporting" because all studies reported the outcomes pre-specified in the methods. All studies were assessed as "low risk" for "other bias", which refers to the inappropriate influence of funders.

\section{Publication bias}

Asymmetrical funnel plots and significant Egger's test ( $\mathrm{t}=3.88, P<0.01)$ indicated potential publication bias in favor of more positive results for the smaller studies (Fig. 5).

\section{Quality of evidence}

Owing to defects in study design and the risk of publication bias, the quality of evidence was downgraded to low according to the GRADE system (Table 4).

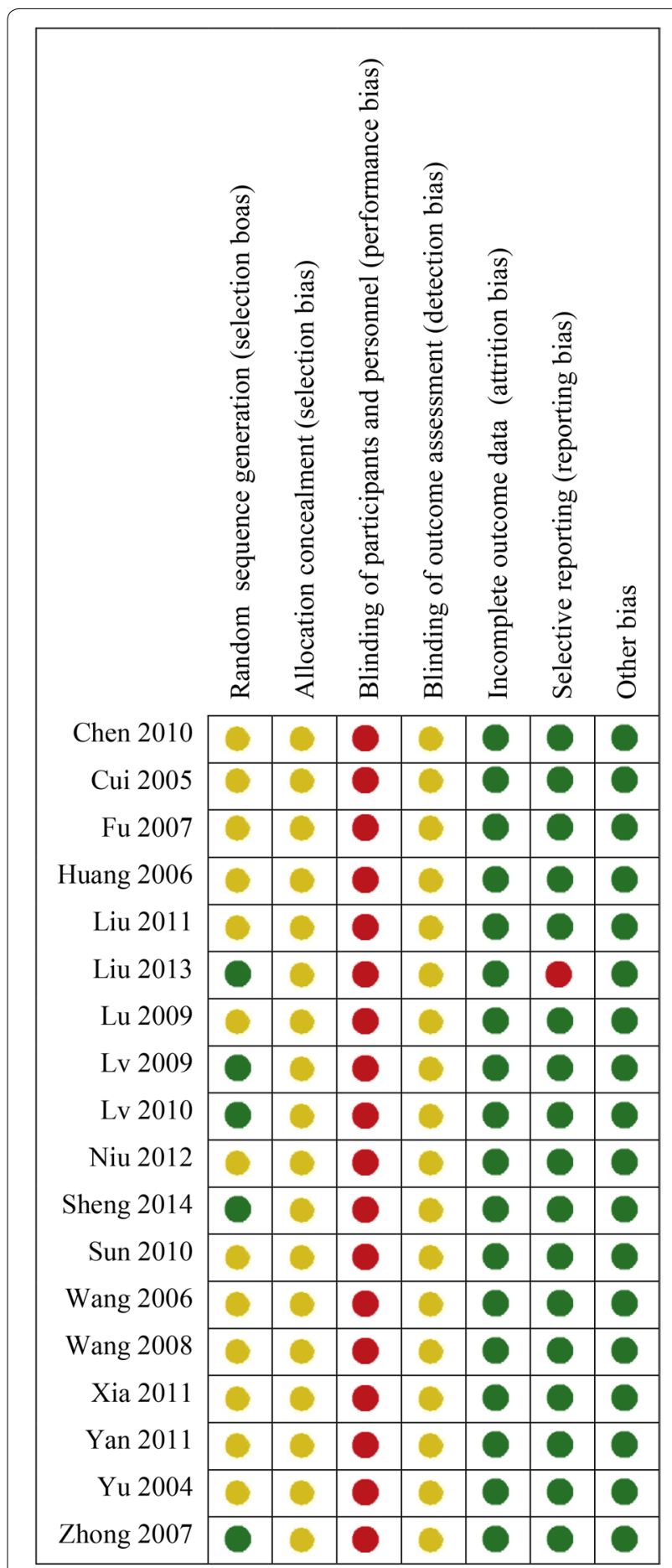

Fig. 4 Risk of bias assessment results. Green circle for low risk of bias, red circle for high risk of bias, yellow circle for unclear risk of bias

\section{Discussion}

Efficacy

The combination of oral CHM and NB-UVB was more effective than NB-UVB alone in achieving PASI-60 


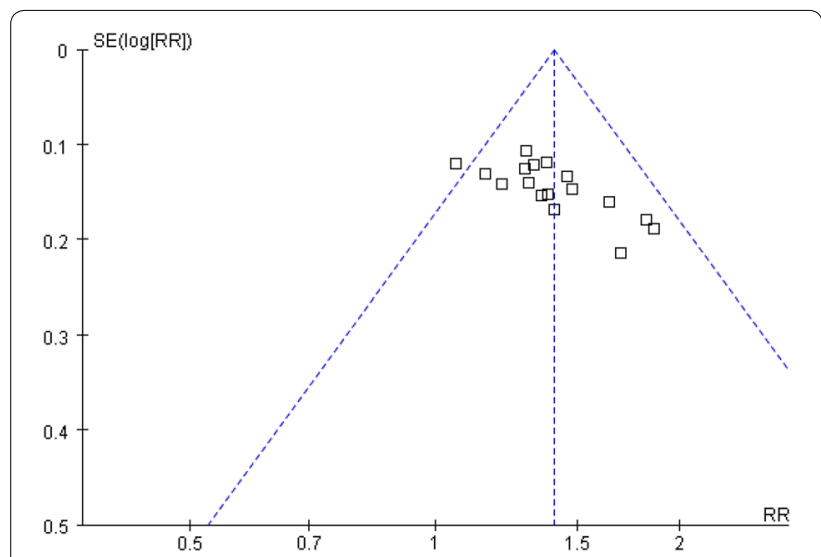

Fig. 5 Funnel plot of the 17 studies that reported PASI-60

after 4-12 weeks of treatment $(\mathrm{RR}=1.40,95 \% \mathrm{CI}$ $1.30-1.50)$ with low heterogeneity $\left(\mathrm{I}^{2}=4 \%\right)$. The estimated NNT to have one achieve PASI-60 with 4-12 weeks treatment was around five. These results were consistent with a systematic review of $\mathrm{CHM}$ bath plus phototherapy for psoriasis vulgaris $(\mathrm{RR}=1.25$, $95 \%$ CI 1.15-1.36) [7].

\section{Adverse events}

Skin reactions such as pruritus and erythema were the most commonly reported AEs following combination treatment with oral $\mathrm{CHM}$ plus $\mathrm{NB}-\mathrm{UVB}$, and were the same as for NB-UVB phototherapy [8]. The combination treatment might lower incidence rates of NB-UVB-associated AEs after 8-12 weeks of treatment. Occasional mild gastrointestinal reactions $(2.87 \%)$ and rare liver function impairment $(0.68 \%)$ were reported in the combination group. All gastrointestinal reactions associated with oral CHM administration were transient and required no additional treatment. Liver function impairment presented as elevated liver enzymes. The studies did not discuss whether the impairment was caused by oral CHM administration, but three of the five participants returned to normal following a reduction in the dosage of CHMs, which suggested that the liver function impairment might be associated with the oral CHMs.

Overdose might be a factor leading to AEs. The dosages of ten herbs, Polygonum multiflorum (he shou wu), R. glutinosa, S. miltiorrhiza, Dictamnus dasycarpus (bai xian pi), Curcuma zedoaria (e zhu), Sophora japonica (huai hua), Scolopendra (wu gong), Bombyx batryticatus

Table 4 Summary of findings

\begin{tabular}{|c|c|c|c|c|c|}
\hline \multicolumn{6}{|c|}{ Oral CHM + NB-UVB compared to NB-UVB for psoriasis vulgaris } \\
\hline \multicolumn{6}{|c|}{ Participant or population: participants with psoriasis vulgaris } \\
\hline \multicolumn{6}{|c|}{ Settings: hospitals in China } \\
\hline \multicolumn{6}{|c|}{ Intervention: oral CHM + NB-UVB } \\
\hline \multicolumn{6}{|l|}{ Comparison: NB-UVB } \\
\hline \multirow[t]{3}{*}{ Outcomes } & \multicolumn{2}{|c|}{ Illustrative comparative risks (95 \% Cl) } & \multirow{3}{*}{$\begin{array}{l}\text { Relative effect } \\
(95 \% \mathrm{Cl})\end{array}$} & \multirow{3}{*}{$\begin{array}{l}\text { No. of participants } \\
\text { (studies) }\end{array}$} & \multirow{3}{*}{$\begin{array}{l}\text { Quality of the } \\
\text { evidence (GRADE) }\end{array}$} \\
\hline & Assumed risk & Corresponding risk & & & \\
\hline & NB-UVB & Oral CHM + NB-UVB & & & \\
\hline PASI 60 & Study population & & $R R 1.35(1.26-1.45)$ & 1339 (17 studies) & $\oplus \oplus \ominus \ominus l o w^{a, b}$ \\
\hline \multirow[t]{3}{*}{ Follow-up 4-12 weeks } & 591 per 1000 & 797 per $1000(744-857)$ & & & \\
\hline & Moderate & & & & \\
\hline & 633 per 1000 & 855 per $1000(798-918)$ & & & \\
\hline PASI 90 & Study population & & $R R 1.71(1.45-2.01)$ & 1342 (17 studies) & $\oplus \oplus \Theta \Theta$ low \\
\hline \multirow[t]{3}{*}{ Follow-up 4-12 weeks } & 219 per 1000 & 375 per 1000 (318-441) & & & \\
\hline & Moderate & & & & \\
\hline & 233 per 1000 & 398 per $1000(338-468)$ & & & \\
\hline
\end{tabular}

GRADE Working Group grades of evidence

High quality: further research is very unlikely to change our confidence in the estimate of effect

Moderate quality: further research is likely to have an important impact on our confidence in the estimate of effect and may change the estimate

Low quality: further research is very likely to have an important impact on our confidence in the estimate of effect and is likely to change the estimate

Very low quality: we are very uncertain about the estimate

a Blindness of participants and investigators is "high risk" in all studies

b Asymmetrical funnel plot and result of Egger's test indicated potential publication bias 
(jiang chan) and Periostracum serpentis (she tui), prescribed in the included studies, exceeded the dosages recommended in the China Pharmacopoeia 2010 [29]. The latest information bulletin on drug adverse reactions published by the China Food and Drug Administration reported that $P$. multiflorum and products containing this plant might be associated with liver function impairment [30], and that overdose and long-term usage could increase the risk of adverse reactions.

\section{Potential mechanisms of action of oral CHM combined with NB-UVB \\ Anti-psoriatic actions of NB-UVB}

Suppression of epidermal hyperproliferation by inhibition of keratinocyte nuclear DNA synthesis is an important mechanism of action of UVB in the treatment of psoriasis [31]. UVB also has anti-inflammatory effects, with inhibition of $\mathrm{T}$ cell activation, induction of $\mathrm{T}$ cell apoptosis and reduction of the release of a variety of proinflammatory cytokines [6].

\section{Anti-psoriatic actions of herbal medicines}

Extracts, compounds or both, of all of the five herbs commonly used in multiple studies, exhibited antiinflammatory effects [32]. In a guinea pig ear model of a psoriasis-like lesion induced by propranolol, parakeratosis pathological changes were significantly alleviated and epidermal proliferation was inhibited by catapol treatment (a major compound of R. glutinosa) [33], but an extract of $R$. glutinosa did not show an anti-proliferative effect on human epidermal keratinocytes ( $\mathrm{HaCaT}$ cells) in vitro [34]. S. miltiorrhiza showed effects on inflammation, proliferation and angiogenesis [35]. In psoriasis-like lesions in guinea pig ears induced by topical propranolol, injection of angelica polysaccharide (a compound in A. sinensis) increased keratinocyte apoptosis [36] and decreased the expression level of proliferating cell nuclear antigen [37]. S. glabra exhibited anti-inflammatory effects and antitumor effects, and inhibited cellular immunity [38]. Anti-proliferative effects of S. glabra were observed in an in vivo study using a mouse vaginal epithelium model and a mouse tail model [39], but there was a negative result observed in an in vitro study using the $\mathrm{HaCaT}$ cell line [34]. Glycyrrhetinic acid (a compound in G. uralensis) had a dose-dependent anti-proliferative effect [40].

Synergetic interaction between NB-UVB and herbal medicine Psoralen in combination with NB-UVB was more effective than NB-UVB alone [41]. Herbs with a high content of psoralen such as Psoralea corylifolia (bu gu zhi) [42] were not used in the included studies. Saposhnikovia divaricata (fang feng), which was used in three studies, contains psoralens such as 5-methoxypsoralen [43]. A. sinensis, which is used in 11 studies, and other Angelica species contain coumarins with photosensitising effects [44]. However, studies are required to examine the effects of combining coumarin-containing herbs and NB-UVB.

The combination treatment reduced the incidence of NB-UVB-induced AEs. Several herbal medicines showed protective effects against UVB-induced skin damage and can relieve pruritus. Survival of normal $\mathrm{HaCaT}$ cells pretreated with a water extract of S. miltiorrhiza prior to UVB exposure was significantly higher than that of cells without pre-treatment [45]. In addition, S. miltiorrhiza showed anti-inflammatory effects by reducing secretion of TNF- $\alpha$ and IL-1 $\beta$. Similar results were found in research on Scutellaria baicalensis (huang qin) and Ligusticum chuanxiong (chuan xiong) [46]. Licoflavone and glycyrrhizin (compounds from G. uralensis), exhibited photo-protective effects on $\mathrm{HaCaT}$ cells irradiated by UVB radiation [47], and UV protective effects for glycyrrhizin and extracts of S. baicalensis and S. divaricata were reported [48]. In a guinea pig foot pruritus model induced by histamine phosphate treatment, catapol (a compound in R. glutinosa) significantly relieved pruritus [33].

\section{Strengths and limitations of this meta-analysis}

The 17 studies included in the meta-analysis were comparable in that they assessed a CHM combined with NBUVB compared with NB-UVB treatment alone, with the difference in PASI score as an outcome. Although the meta-analysis results for the subgroups and sensitivity analyses tended to be consistent with those for the total pool, in terms of the quality of the evidence, a number of issues led to this being downgraded to low. Because none of the studies used a placebo for the CHM in the control arm, blinding of participants could not be achieved. Consequently, any placebo effect could not be distinguished from a genuine add-on biological therapeutic effect. Sequence generation was unclear in 13 studies; we are not sure whether these studies were truly randomized. The asymmetrical funnel plot showed that the smaller studies reported more positive results, suggesting that there might be a publication bias.

Other issues that may impact upon the interpretability of the results were variability in the severity of psoriasis in the participants, the duration and dosage of the NB-UVB and CHM treatments, and variability in the composition and quality of the CHMs. It was not feasible to explore all of these issues owing to limitations in the available data, but the sub-group analyses that were undertaken were consistent with the PASI-60 results for the total pool. Additionally, all included studies were conducted in China and the samples were restricted to 
Chinese populations, which might limit the generalization of the results. Adverse events were not reported during the follow-up periods and the criteria for relapse rate during follow up were not well defined. As such, the long-term efficacy of and the adverse events associated with the combination treatment could not be evaluated in this study.

\section{Implications for clinical practice and further research}

The results of this study suggested that, in clinical psoriasis vulgaris treatment, the three most frequently used herbs, $R$. glutinosa, A. sinensis, S. glabra, could be considered the core herbs for treating participants undergoing NB-UVB treatment. The dose for each herb should be in strict compliance with the China Pharmacopoeia and regular monitoring of liver function is needed during treatment.

In further research, the disease severity of psoriasis participants should be clearly reported, and a stratified (by disease severity) analysis should be performed. A placebo for the CHM should be used in the control group to enable effective blinding. For alignment with international studies, PASI-50 and PASI-75 should be reported. Remission or relapse should be clearly defined and reported to evaluate long-term effectiveness. In addition, whether combination treatment could reduce the number of treatment sessions and/or the cumulative dose of NB-UVB should be investigated. Furthermore, a study on the long-term safety of this combination treatment is needed.

\section{Conclusion}

Orally administered CHM combined with NB-UVB showed improved efficacy for treating psoriasis vulgaris but quality of evidence was low.

\section{Additional file}

Additional file 1: Table S1. Herbs used in the included studies.

\begin{abstract}
Abbreviations
CHM: Chinese herbal medicine; RCT: randomized controlled trial; PUVA: psoralen plus ultraviolet A; NB-UVB: narrowband ultraviolet B; BB-UVB: broad-band ultraviolet $B ;$ CNKI: China National Knowledge Infrastructure; CBM: Chinese BioMedical Literature; CQVIP: Chinese Science Journals Full Text; PASI: Psoriasis Area Severity Index; BSA: body surface area; AE: adverse event; GRADR: the grades of recommendation, assessment, development and evaluation; RR: risk ratio; MD: mean difference; Cl: confidence interval; ITT: intention-to-treat; NNT: number needed to treat; $\mathrm{HaCaT}$ cell: human epidermal keratinocyte; TNF- $\alpha$ : tumor necrosis factor $\alpha$; IL-1 $\beta$ : interleukin-1 $\beta$; UV: ultraviolet.
\end{abstract}

\section{Authors' contributions}

$C L$ and $C X$ conceived the study. LY, CZ and JY searched the databases and performed risk of bias assessment. JY, CZ, XG, BM and AZ performed data analysis. LY and CZ wrote the manuscript. All authors read and approved the final manuscript.

\section{Author details}

1 Evidence Based Medicine and Clinical Research Service Group, Guangdong Provincial Hospital of Chinese Medicine, Guangzhou, China. ${ }^{2}$ Department of Dermatology, Guangdong Provincial Hospital of Chinese Medicine, Guangzhou, China. ${ }^{3}$ The 2nd Clinical College of Guangzhou University of Chinese Medicine, Guangzhou, China. ${ }^{4}$ Guangdong Provincial Academy of Chinese Medical Sciences, Guangzhou 510120, China. ${ }^{5}$ China-Australia International Research Centre for Chinese Medicine, School of Health Sciences, RMIT Health Innovations Research Institute, RMIT University, PO Box 71, Bundoora, Melbourne, VIC 3083, Australia.

\section{Acknowledgements}

This study was mainly funded by a grant from the Guangdong Provincial Science and Technology Department and the Guangdong Provincial Academy of Chinese Medical Sciences (GPACMS) (Grant No. 2012A032500009). The study is partially supported by a grant from the International Science and Technology Cooperation Project of the Ministry of Science and Technology of China (Grant No. 2012DFA31760) and the China-Australia International Research Center for Chinese Medicine, funded by the Guangdong Provincial Academy of Chinese Medical Sciences and Guangdong Provincial Hospital of Chinese Medicine (GPACMS and GPHCM), Guangdong China and RMIT University, Australia.

\section{Compliance with ethical guidelines}

\section{Competing interests}

The authors declare that they have no competing interests.

Received: 30 July 2014 Accepted: 17 September 2015

Published online: 26 September 2015

\section{References}

1. Cimmino MA. Epidemiology of psoriasis and psoriatic arthritis. Reumatismo. 2007:59(Suppl 1):19-24.

2. Menter A, Gottlieb A, Feldman SR, Van Voorhees AS, Leonardi CL, Gordon KB, Lebwohl M, Koo JY, Elmets CA, Korman NJ, Beutner KR, Bhushan R. Guidelines of care for the management of psoriasis and psoriatic arthritis: section 1. Overview of psoriasis and guidelines of care for the treatment of psoriasis with biologics. J Am Acad Dermatol. 2008;58:826-50.

3. Archier E, Devaux S, Castela E, Gallini A, Aubin F, Le Maitre M, Aractingi S, Bachelez H, Cribier B, Joly P, Jullien D, Misery L, Paul C, Ortonne JP, Richard MA. Carcinogenic risks of psoralen UV-A therapy and narrowband UV-B therapy in chronic plaque psoriasis: a systematic literature review. J Eur Acad Dermatol Venereol. 2012;26(Suppl 3):22-31.

4. Almutawa F, Alnomair N, Wang Y, Hamzavi I, Lim HW. Systematic review of UV-based therapy for psoriasis. Am J Clin Dermatol. 2013;14:87-109.

5. Archier E, Devaux S, Castela E, Gallini A, Aubin F, Le Maitre M, Aractingi S, Bachelez H, Cribier B, Joly P, Jullien D, Misery L, Paul C, Ortonne JP, Richard MA. Efficacy of psoralen UV-A therapy vs. narrowband UV-B therapy in chronic plaque psoriasis: a systematic literature review. J Eur Acad Dermatol Venereol. 2012;26(Suppl 3):11-21.

6. Nast A, Boehncke WH, Mrowietz U, Ockenfels HM, Philipp S, Reich K, Rosenbach T, Sammain A, Schlaeger M, Sebastian M, Sterry W, Streit V, Augustin M, Erdmann R, Klaus J, Koza J, Muller S, Orzechowski HD, Rosumeck S, Schmid-Ott G, Weberschock T, Rzany B. S3-guidelines for the treatment of psoriasis vulgaris Update 2011. J Dtsch Dermatol Ges. 2011;9(Suppl 2):S1-104.

7. Yu JJ, Zhang CS, Zhang AL, May B, Xue CC, Lu C. Add-on effect of Chinese herbal medicine bath to phototherapy for psoriasis vulgaris: a systematic review. Evid Based Complement Altern Med. 2013;2013:673078.

8. Cui X. Clinical observation of NB-UVB combined Chinese herbal Medicine in treatment of psoriasis vulgaris [Chinese]. Thesis, Nanjing University of Traditional Chinese Medicine; 2005.

9. Higgins JGS. Cochrane handbook for systematic reviews of interventions version 5.1.0. The Cochrane Collaboration, 2011.

10. Chen R. Research of the combination of Huayu Xiaoyin tang and UVB impact on efficacy and hemorheology for psoriasis patients with blood stasis pattern [Chinese]. Thesis, Shandong University of Traditional Chinese Medicine; 2010. 
11. Fu S, Wang W, Li M. Effectiveness observation of the combination of Yangxue huoxue tang and NB-UVB for plaque psoriasis [Chinese]. Zhong Guo Ma Feng Bing Xue Za Zhi. 2007;23:824-5.

12. Huang DH, Li YS, Huang Y, Tan ZK. Effectiveness of the combination of Huobahuagen pian and NB-UVB in treatment of psoriasis vulgaris [Chinese]. Zhong Guo Zhong Xi Yi Jie He Pi Fu Xing Bing Xue Za Zhi. 2006;5:104-5.

13. Liu H, Sheng G. Effectiveness of Tuiyin tang combined with NB-UVB in treatment of psoriasis [Chinese]. Zhong Guo Xian Dai Ying Yong Yao Xue. 2013;30:1131-5.

14. Liu Y, Yan L. Clinical observation on combination of Xiaoyin Granules with NB-UVB for reducing relapse rate of psoriasis [Chinese]. Lin Chuang Pi Fu Ke Za Zhi. 2011;40:767-9.

15. Lu M, Ye J, Zhang Y, Peng K. YL S: Clinical observation of NB-UVB combined Chinese herbal Medicine in treatment of psoriasis vulgaris patients with blood dryness pattern [Chinese]. Si Chuan Zhong Yi. 2009;27:102-3.

16. Lv H, Zheng W, Yan X. Clinical observation of the combination of Liangxue tongluo tang and NB-NVB in treatment of psoriasis vulgaris [Chinese]. Zhong Guo Zhong Xi Yi Jie He Za Zhi. 2010;30:555-6.

17. LV P. Effectiveness of the combination of Keyin wan and NB-UVB in treatment of psoriasis vulgaris [Chinese]. Zhong Guo Pi Fu Xing Bing Xue Za Zhi. 2009;23:539-40.

18. Niu R. The clinical and experimental study on blood-heat syndrome of psoriasis vulgaris treating with NB-UVB combined with Liang Xue Xiao Feng decoction [Chinese].Thesis, Tianjin Medical University; 2012.

19. Sheng $G$, Xie Y, Liu H. Effect of Tuiyin decoction combined with NB-UVB in the treatment of psoriasis vulgaris and the influence on expression levels of related cytokines in peripheral blood [Chinese]. Zhong Guo Yi Yuan Yao Xue Za Zhi. 2014;34:576-80.

20. Sun W, Yan H, Sun Z, Zhao L. Effectiveness observation of the combination of Huayu baibi jiaonang and NB-UVB for psoriasis [Chinese]. Guang Ming Zhong Yi. 2010;25:2013-4.

21. Xia X, Zhang WX. Effectiveness of the combination of Liangxue xiaofeng tang and NB-UVB in treatment of psoriasis vulgaris [Chinese]. Shi Yong Zhong Yi Yao Za Zhi. 2011;27:371-2.

22. Wang G, Hou X, Liu W, Jin L. Effectiveness of the combination of Shendi chongji and NB-UVB in treatment of psoriasis patients with blood heat pattern [Chinese]. Zhong Guo Zhong Xi Yi Jie He Pi Fu Xing Bing Xue Za Zhi. 2008;7:98-9.

23. Wang L. Mechanism and dose of radiation in combination treatment for psoriasis vulgaris [Chinese]. Thesis, Dalian Medical University; 2006.

24. Yan $\mathrm{H}, \mathrm{Du} \mathrm{Q}$, Song A. Effectiveness observation of the combination of Wushe jiedu wan and NB-UVB for psoriasis vulgaris [Chinese]. Zhong Guo She Qu Yi Sheng. 2011;13:127.

25. Yu L. Clinical observation of the combination of Goeckerman therapy and Chinese herbal Medicine in treatment of psoriasis [Chinese]. Zhong Guo Pi Fu Xing Bing Xue Za Zhi. 2004;18:31.

26. Zhong J, Lu CJ, He WQ, Liang YM, Xuan GW. The combination of NBUVB and Xiaoyin keli for psoriasis patients with blood deficiency and wind dryness pattern [Chinese]. Ling Nan Pi Fu Xing Bing Xue Za Zhi. 2007:14:98-100.

27. Dermatology Committee of People's Liberation Army Association of. Traditional Chinese medicine: consensus on diagnosis and treatment of Chinese integrative medicine for psoriasis vulgaris. Chin J Dermatol. 2009;8:328.

28. Carlin CS, Feldman SR, Krueger JG, Menter A, Krueger GG. A 50\% reduction in the Psoriasis Area and Severity Index (PASI 50) is a clinically significant endpoint in the assessment of psoriasis. J Am Acad Dermatol. 2004;50:859-66.

29. Commission CP. China pharmacopoeia. Beijing: China Medical Science Press; 2010
30. China Food and Drug Administration. Pay attention to liver impairments induced by orally administration with he shou wu and products contained he shou wu. Inf Bull Drug Advers React. 2014;61.

31. Ibbotson SH, Bilsland D, Cox NH, Dawe RS, Diffey B, Edwards C, Farr PM, Ferguson J, Hart G, Hawk J, Lloyd J, Martin C, Moseley H, McKenna K, Rhodes LE, Taylor DK. An update and guidance on narrowband ultraviolet B phototherapy: a British Photodermatology Group Workshop Report. Br J Dermatol. 2004;151:283-97.

32. Tse TW. Use of common Chinese herbs in the treatment of psoriasis. Clin Exp Dermatol. 2003;28:469-75

33. Kuang Y. Study on the dynamic accumulation of catolpol in fresh leaves of Rehmannia glutinosa and its anti-psoriasis affect [Chinese]. Thesis, Academy of Military Medical Sciences; 2009.

34. Tse WP, Che CT, Liu K, Lin ZX. Evaluation of the anti-proliferative properties of selected psoriasis-treating Chinese medicines on cultured $\mathrm{HaCaT}$ cells. J Ethnopharmacol. 2006;108:133-41.

35. Deng S, May BH, Zhang AL, Lu C, Xue CC. Phytotherapy in the management of psoriasis: a review of the efficacy and safety of oral interventions and the pharmacological actions of the main plants. Arch Dermatol Res. 2014;306:211-29.

36. Jing $H$, Sheng $W$. Influence of Angelica polysaccharide on apoptosis in keratinocytes of psoriasis-like lesions of guinea pigs [Chinese]. Med J Wuhan Univ. 2006;27:377-80.

37. Jing $H$, Sheng $W$, Duan D. Influence of angelica polysaccharide on the expression levels of PCNA in psoriasis-like lesions of guinea Pigs [Chinese]. Zhong Guo Pi Fu Xing Bing Xue Za Zhi. 2008;22:11-3.

38. Jiang S. Review of modern research on Smilax glabra and Smilax china. Intern Med. 2010;5:191-5.

39. Liu X, Sun X, Qi X, Lin X. Influence on epithelial cell proliferation, epidermal cell differentiation and plasma endothelin-1 with 20 Chinese herbal medicine intragastric administration in mice. Chinese Journal of Dermatology. 2001;34:35-6.

40. Xie F. Mechanism of inhibitive effect of glycyrrhetinic acid on proliferation of HaCat cell induced by epidermal growth factor [Chinese]. Thesis, Zhejiang University of Traditional Chinese Medicine; 2009.

41. Khurshid K, Haroon TS, Hussain I, Pal SS, Jahangir M, Zaman T. Psoralenultraviolet A therapy vs. psoralen-ultraviolet B therapy in the treatment of plaque-type psoriasis: our experience with fitzpatrick skin type IV. Int J Dermatol. 2000;39:865-7.

42. Ding J, Zhang Q, Zhang L. Content determination for psoralen and isopsoralen in Psoralea corylifolia from different regions. Zhong yao cai. 2004;27:817-8.

43. Zhou J, Xie G, Yan X. Encyclopedia of traditional chinese medicines: molecular structures, pharmacological activities, natural sources and applications. New York: Springer; 2011.

44. Koo J, Desai R. Traditional Chinese medicine in dermatology. Dermatol Ther. 2003;16:98-105.

45. Deng L, Zhang L, Bai Q, Chen G. Photo-protection effect of Salvia miltiorrhiza and fructus lycii on human keratinocytes. J Jiangxi Univ TCM. 2007;19:61-3.

46. Luo D, Min W, Lin X, Wang S. Photo-protective effect of hydroxychloroquine and Chinese herbal medicines on human keratinocytes damaged by ultraviolet irradiation. Chin J Aesthet Med. 2003;12:355-8.

47. Yang $L$. The protective effect and mechanism of licoflavone and glycyrrhizin on HaCaT cells irradiated by UVB [Chinese]. Thesis, Lanzhou University; 2011

48. Kato T, Hino S, Horie N, Shimoyama T, Kaneko T, Kusama K, Sakagami H. Anti-UV activity of Kampo medicines and constituent plant extracts: reevaluation with skin keratinocyte system. In Vivo. 2014;28:571-8. 